\title{
ERROR RECOGNITION IN VELOCITY MODEL BUILDING FOR PRESTAK KIRCHOFF DEPTH MIRATION USING RMO ANALYSIS
}

DOI: http://dx.doi.org/10.18509/GBP.2015.14

UDC: 550.344.094.5:550.83.088

\author{
Dr. Maya Grigorova \\ University of Mining and geology “St. Ivan Rilski”, Bulgaria
}

\begin{abstract}
The main purpose in seismic investigations is the correct illustration of geology in the study area. In seismic processing even small variations can have a significant impact, hiding the real signature or introducing artifacts. Velocity errors can be critical for accurate seismic representations of the modeled geology and ignoring them can damage the structural interpretation of the study area. As far as the velocity model is representative of the subsurface, Kirchoff pre-stack depth migration (PSDM) is a reasonable representation of the modeled geology.
\end{abstract}

Keywords: Velocity model, RMO, seismic tomography, velocity errors, PSDM.

\section{INTRODUCTION}

Building a depth-velocity model is an important step in the PSDM workflow. Migration needs an accurate velocity model to fully focus reflections and correctly position reflectors in space. Pre-Stack Depth Migration requires an accurate interval velocity model to achieve optimum results. The process requires utilisation of RMS velocities from the seismic data. The determination of an accurate migration velocity in time domain is a crucial step in seismic imaging. In case migration velocity is unappropriated then significant changes in geology can occur in the depth migrated data.

The effect of velocity on depth migration is significant since the velocity errors leads to improper positioning and poor focusing migrated reflection events. Here is described relationship between inaccurate input velocities and depth positional errors on the depth migrated section. It is demonstrated that even small velocity errors are problematic, especially with pre-stack depth migration.

\section{BUILDING VELOCITY MODEL FOR PRESTACK KIRCHOFF DEPTH MIGRATION}

The fundamental principle underlying velocity model building is that the correct velocity must accurately explain the relative time delays between reflections that are originated from the same interface in the subsurface.

On a seismic record the reflection from a flat horizontal layer appears curved. This is because the travel path to a detector at some distance $\mathrm{x}$ is longer than the travel path to a detector at the source. The vertical two-way travel time $\left(\mathrm{T}_{0}\right)$ to and from the reflector is equal to twice the depth of the reflector (D) divided by the average velocity $\left(\mathrm{V}_{\text {ave }}\right)$ between the surface and the reflector:

$T_{0}=\frac{2 D}{V_{c p}}$ 
The difference between the reflection time at $\mathrm{x}$ and the reflection time at zero distance $\left(\mathrm{T}_{0}\right)$ is called "normal moveout" $(\mathrm{NMO})(\Delta \mathrm{T})$ :

$T=T_{0}+\Delta T$

In this way with increasing the offset the difference in the arrival time become bigger (Figure 1).

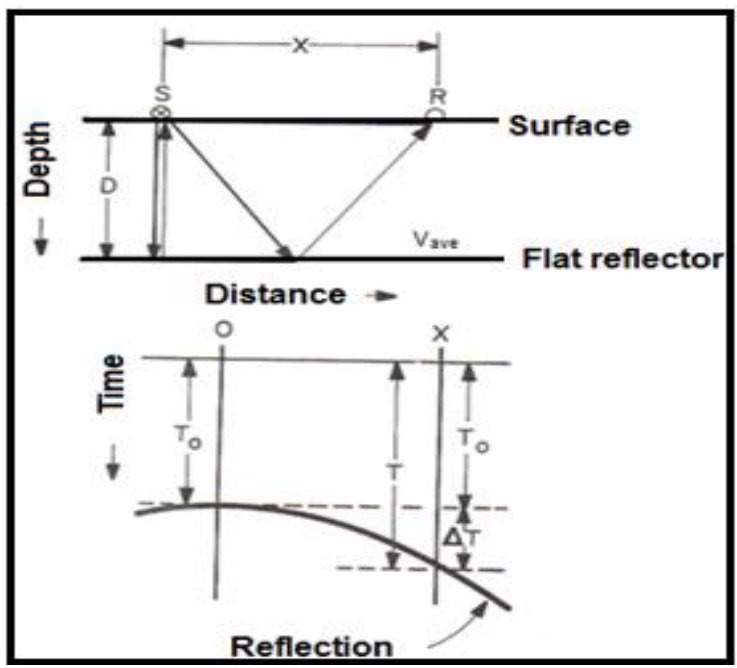

Figure 1. Vertical wave path from source to reflection surface and reverse to detector on the ground

The relationship between arrival time and offset is hyperbolic and it is the principal criterion that a geophysicist uses to decide whether an event is a reflection or not.

The source projection $S$ ', so that the horizontal distance $x$ and the travel paths $T_{0} V_{\text {ave }}(S S$ ' $=\mathrm{T}_{0} \mathrm{~V}_{\mathrm{ave}}$ ) and $\mathrm{TV}_{\mathrm{ave}}$ form a right triangle which can be solved by the Pythagorean Theorem [1]:

$T^{2} V_{\text {ave }}=T_{0}^{2} V_{\text {ave }}^{2}+x$

To obtain normal moveout $(\Delta \mathrm{T})$ we substitute $T=T_{0}+\Delta T$ :

$\left(T_{0}+\Delta T\right)^{2} V_{\text {ave }}^{2}=T_{0}^{2} V_{\text {ave }}^{2}+x^{2}$

$\left(T_{0}+\Delta T\right)^{2}=T_{0}^{2}+\frac{x^{2}}{V_{a v e}^{2}}$

$T_{0}+\Delta T=\sqrt{T_{0}^{2}+\frac{x^{2}}{V_{\text {ave }}^{2}}}$

$\Delta T=\sqrt{T_{0}^{2}+\frac{x^{2}}{V_{a v e}^{2}}}+T_{0}$

The large majority of velocity estimation methods are based on the analysis of the kinematic of reflections. The kinematic corrections are analysed directly in the data domain when both the geological structure and the velocity model are simple. When either the reflector geometry is complex, or the wavefronts are distorted by a rapidly varying velocity function, the analysis of the kinematics is more likely to include errors [2]. Geological knowledge of the subsurface may provide some information on the spatial distribution of propagation velocities, but detailed information can only retrieved from the seismic data themselves. 


\section{POSSIBLE VELOCITY ERRORS IN VELOCITY MODEL BUILDING}

For experimental purposes two velocity models are built:

- Velocity model without modifications (errors);

- Velocity model with reduced velocities in the interval 300-500 cdp;

Velocity field without modification and modified velocity fields are shown on Figure 2.
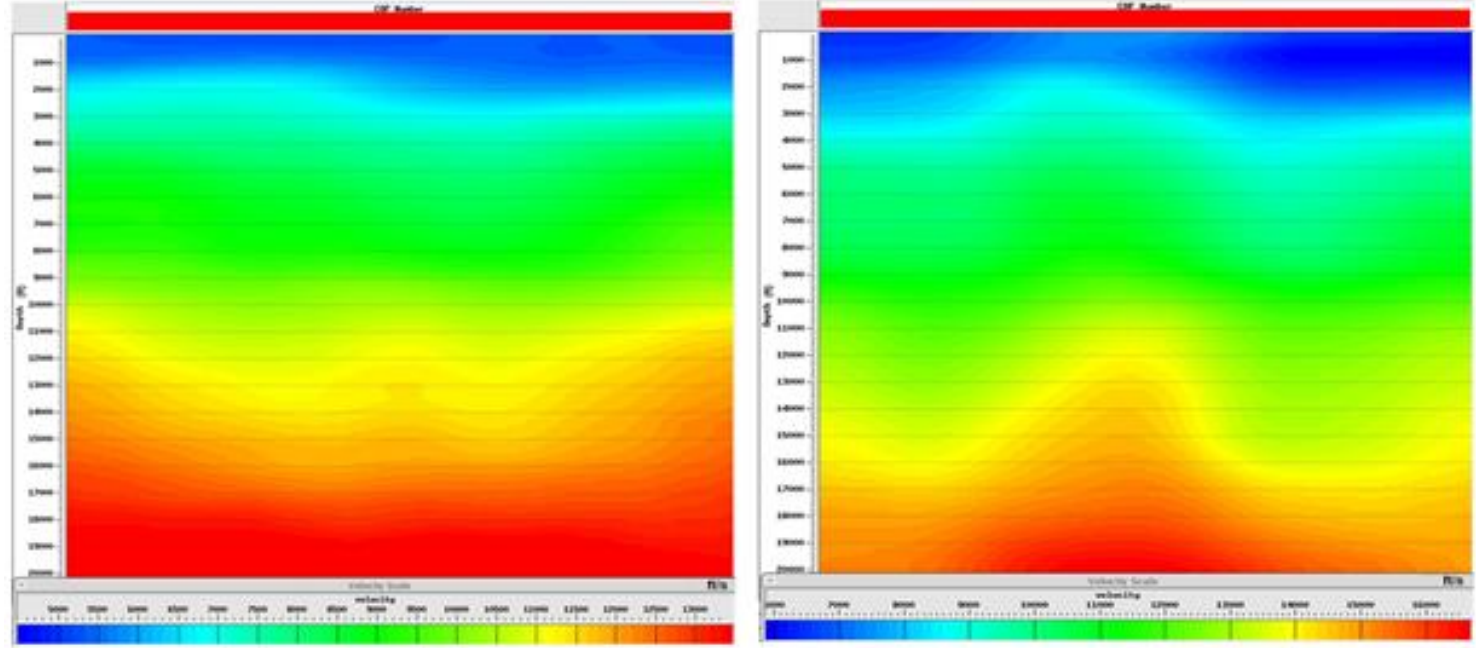

Figure 2. Velocity fields of: velocity model without modifications (errors) and velocity model with reduced velocities in the interval $300-500 \mathrm{cdp}$

The first step in the process is to run the PSDM with an existing interval velocity model in depth domain.
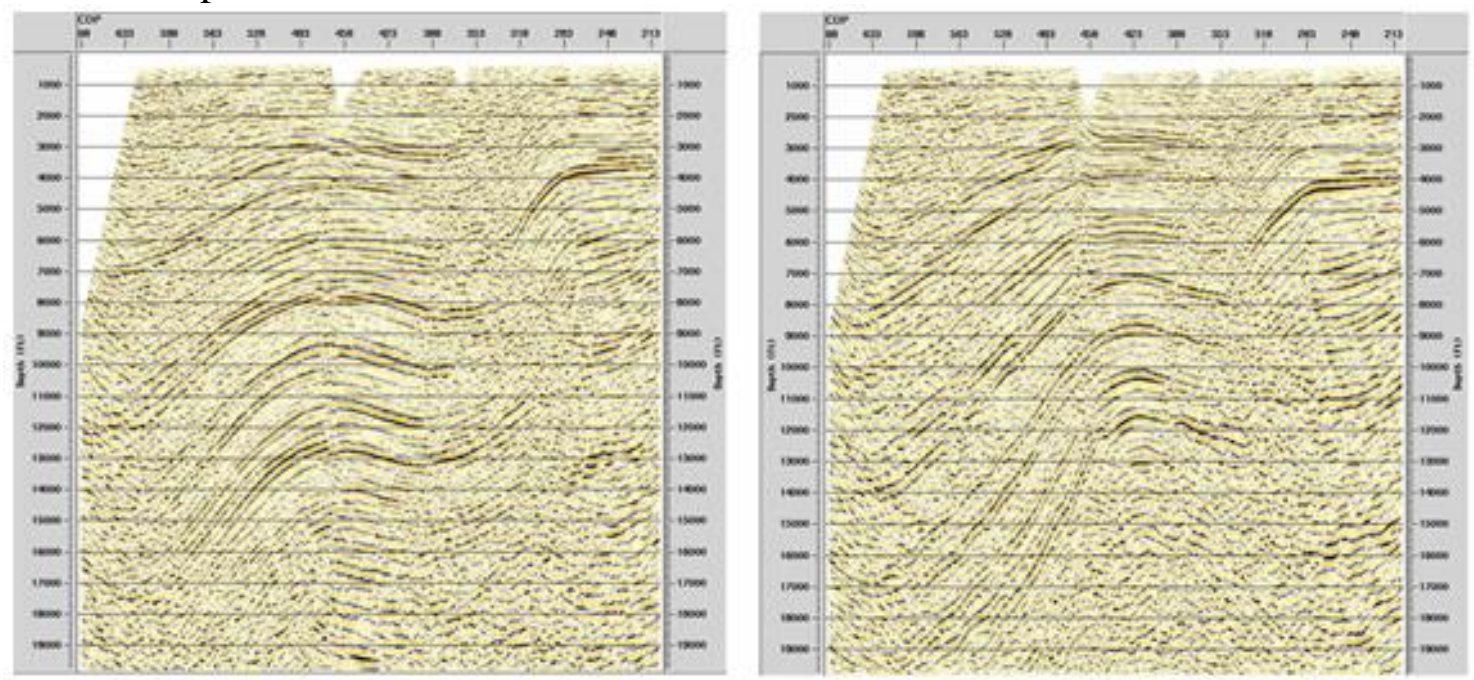

Figure 3. Preliminary PSDM with velocity model without modifications (errors) and velocity model with reduced velocities in the interval $300-500 \mathrm{cdp}$

Once the initial PSDM is complete, it is necessary to be picked the moveout on the depth gathers and the picks are used for input to tomography. Any available geological information is incorporated at this stage to add some constraints to the model. The interval velocity model is then sub-divided into discrete velocity layers which match as closely as 
possible the geological horizons. The velocity model is refined and updated though a number of PSDM iterations using tomographic updates [3].

The depth model reflects how the separation of the subsurface is transformed into formations and indicates which part of the subsurface reflects the respective formation [4].

Conventional velocity analysis methods generally assume flat-layered geology and mild lateral velocity variations. In areas with complex structural geology formations, these methods often fail, and more sophisticated techniques are required.

One of these techniques, seismic tomography, compares observed travel times, measured for each source-receiver pair, with expected travel times, computed by ray tracing through an assumed velocity model. The difference is projected back over the traced ray paths to produce an update to the model [5].
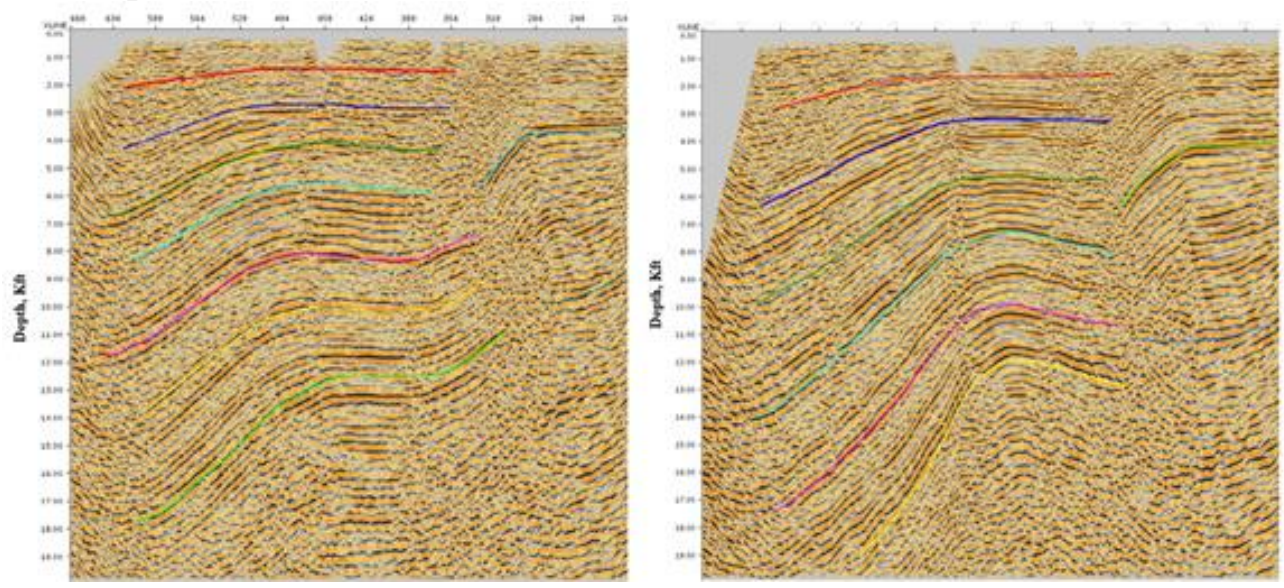

Figure 4. Stacked data after performing PSDM with picked horizons for velocity model without modifications (errors) and velocity model with reduced velocities in the interval $300-500 \mathrm{cdp}$

The tomography uses the ray traced travel time tables, saved from the migration, and the user picks, to construct source-receiver ray paths at the pick locations. The moveout described by the picks is then analysed, in conjunction with the ray paths, to determine the necessary change in velocity which will remove the moveout from the gather [6]. Velocities fields after seismic tomography are shown on figure 5.
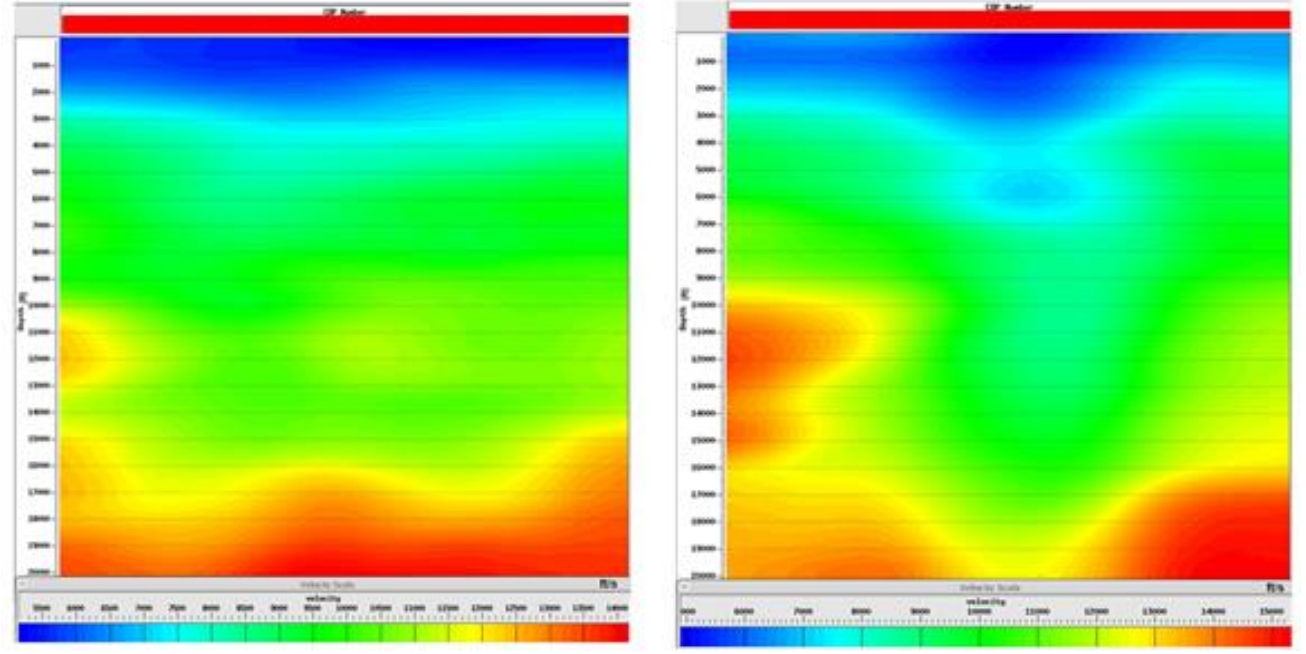

Figure 5. Velocity fields after tomography for velocity model without modifications (errors) and velocity model with reduced velocities in the interval 300-500 cdp 
After building new velocity models using tomography and tracing the ray paths Pre-Stack Kirchhoff Depth migration is performed (Figure 6).
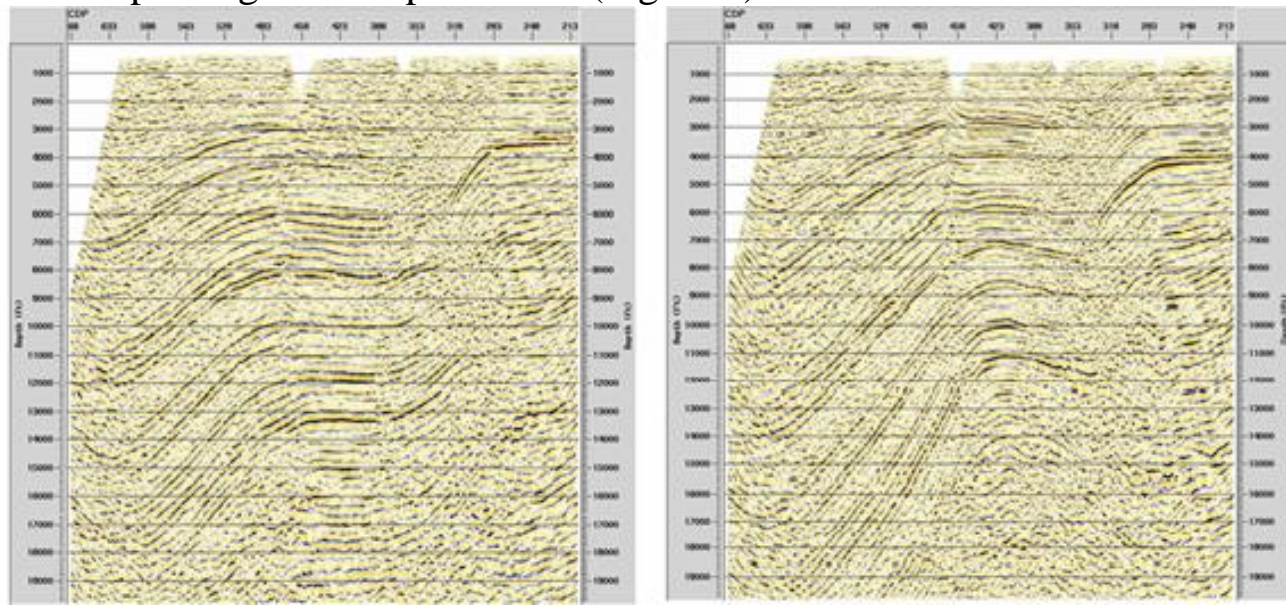

Figure 6. Final stack data after PSDM with velocity model without modifications (errors) and velocity model with reduced velocities in the interval 300-500 cdp

\section{RMO ANALYSIS AFTER PRE-STACK KIRCHOFF DEPTH IGRATION}

After several iterations of PSDM residual moveout is performed of the depth migrated gathers. On figure 7 are shown RMO analysis for both models: velocity model without modifications (errors) and velocity model with reduced velocities in the interval 300-500 cdp. On the figure 7A is observed velocity model without modifications (errors) where it is obvious that residual velocity function (black line) is accurately positioned under the zero point. That means the necessity of residual moveout is insignificant. The velocity model build by tomography with properly chosen kinematic correction can be used successfully for structural interpretation purposes. Otherwise on figure $7 \mathrm{~B}$ is shown velocity model with reduced velocities in the interval $300-500 \mathrm{cdp}$. On the figure is clearly observed that residual velocity function is decline from the zero point and the necessity of negative residual moveout is obvious. This is sure sign that velocity model obtain after tomography does not compensate kinematic moveout. 

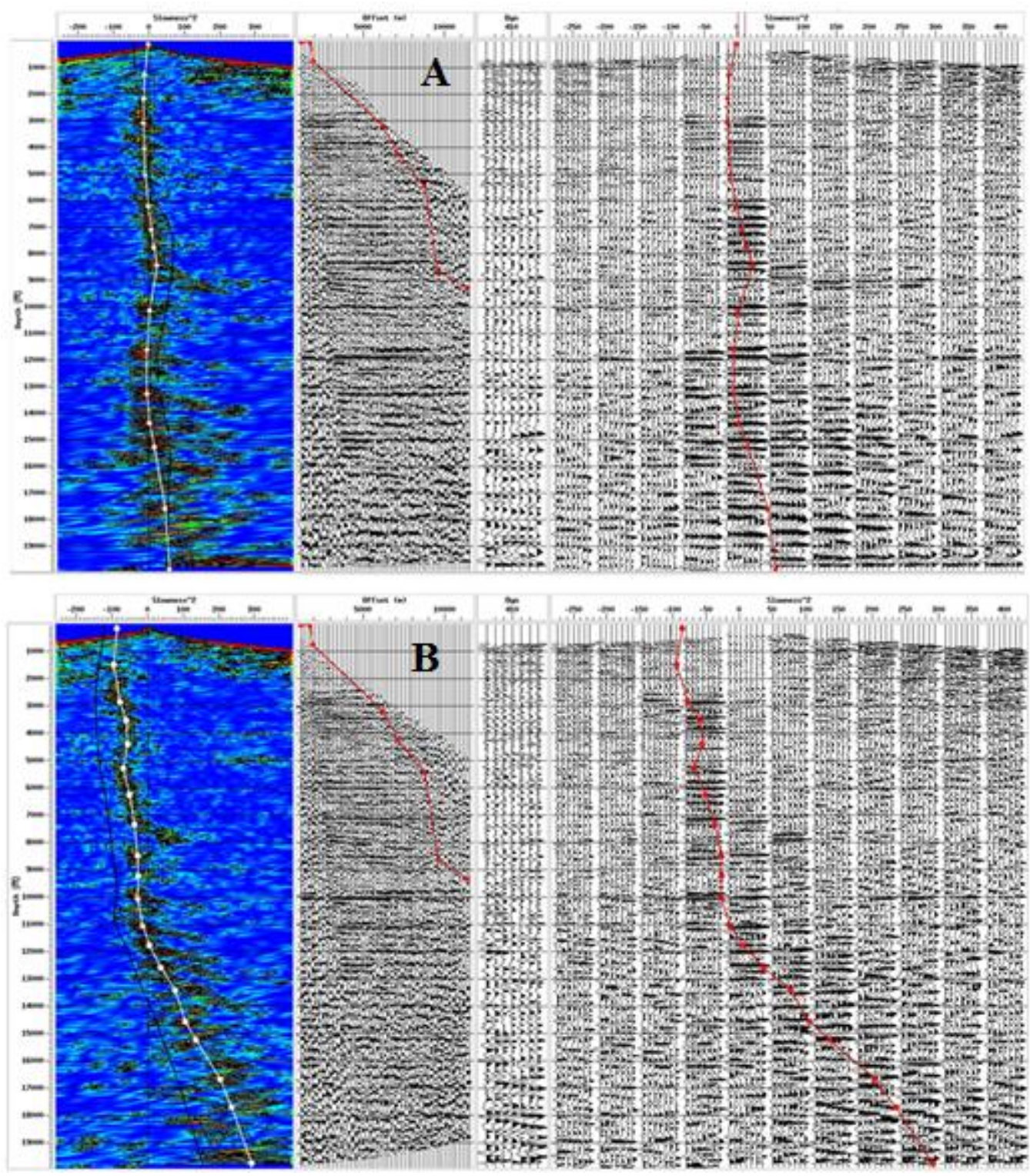

Figure 7. RMO analysis for velocity model without modifications (errors) (top) and velocity model with reduced velocities in the interval $300-500 \mathrm{cdp}$ (bottom)

\section{CONCLUSION}

Based on conducted research following conclusions can be drawn:

- For better depth migration precise velocity model is needed. A careful examination of the seismic velocity analysis technique is key factor for obtaining geologically reasonable pre-stack depth migration;

- Final result from Pre-Stack Depth Migration obtained by the velocity model created by tomography can be used successfully for structural interpretation purposes after careful consideration of RMO corrections;

- Residual velocity analysis is reliable tool for velocity model estimation and for improvement stack conditions of pre-stack depth migrated data. 


\section{REFERENCES}

[1] Grigorova, M., The aim of kinematics in 2D land seismic processing data for enhancing signal - to - noise ratio. Proceedings of XV BMPC, Bulgaria, vol. 2, pp 1260$1262,2013$.

[2] http://sepwww.stanford.edu/sep/biondo/HTML/VelAn.html

[3] Southward D., Oldfield R., Benson C., Pre-stack depth migration on regional 2D seismic surveys. A cost and time effective way of optimising structural detail, Spectrum, 2008

[4] Cameron M., Fomel S., Sethian J., Seismic velocity estimation and time to depth conversion of time-migrated images. SEG International Exposition and 76th Annual Meeting, 6, pp 3113-3128, 2006.

[5] Johannes A. van Trier, Tomographic determination of structural velocities from depth-migrated seismic data, Stanford Exploration Project No 66, pp 5-6, 1990

[6] Tsunami Development Software, 2012, www.tsunamidevelopment.com 
International Scientific Conference GEOBALCANICA 2015 